\title{
Nanoscale
}

RSCPublishing

\section{Fundamental quantum noise mapping with tunnelling microscopes tested at surface structures of subatomic lateral size $\dagger$}

\author{
Markus Herz, Samuel Bouvron, Elizabeta Ćavar, Mikhail Fonin, Wolfgang Belzig \\ and Elke Scheer*
}

We present a measurement scheme that enables quantitative detection of the shot noise in a scanning tunnelling microscope while scanning the sample. As test objects we study defect structures produced on an iridium single crystal at low temperatures. The defect structures appear in the constant current images as protrusions with curvature radii well below the atomic diameter. The measured power

Received 1st May 2013

Accepted 28th July 2013

DOI: $10.1039 / c 3 n r 02216 a$ spectral density of the noise is very near to the quantum limit with Fano factor $F=1$. While the constant current images show detailed structures expected for tunnelling involving $d$-atomic orbitals of Ir, we find the current noise to be without pronounced spatial variation as expected for shot noise arising from statistically independent events.

www.rsc.org/nanoscale

\section{Introduction}

For the development of atomic and molecular size functional electronic building blocks the knowledge of the nature of the electronic transport is crucial. For instance quantum mechanical tunnelling enables phase coherent transport, while the phase information in thermally activated transport processes is randomized. ${ }^{1}$ Shot noise measurements have been proven to be an important tool for revealing electronic interactions and correlations in electronic transport in a variety of systems ${ }^{2}$ ranging from the fractional quantum Hall regime ${ }^{3,4}$ via metallic quantum $\operatorname{dots}^{5}$ and superconducting systems ${ }^{6}$ to atomic contacts $^{7-15}$ including elastic ${ }^{4-7}$ as well as inelastic electronic transport properties. $^{8-15}$ Further promising prospects of tunnelling noise measurements have been demonstrated by superconducting on-chip detection schemes,${ }^{\mathbf{1 6}}$ primary electron thermometry ${ }^{17}$ and dynamical environmental effects on higher cumulants. ${ }^{18}$ The absolute limits of single charge detection by measuring the full counting statistics directly were achieved using a quantum point contact detector of the charge in quantum dots. ${ }^{19-22}$

In the seminal work of Birk et al. ${ }^{5}$ ultra-high resolution of the noise signal has been obtained with a scanning tunnelling microscope (STM) operating under steady conditions, i.e. without scanning. An elegant solution combines direct current mapping with an indirect measurement of the noise by recording the emitted light intensity. ${ }^{23,24}$ To the best of our

Department of Physics, University of Konstanz, 78457 Konstanz, Germany. E-mail: elke.scheer@uni-konstanz.de

$\dagger$ Electronic supplementary information (ESI) available. See DOI: 10.1039/c3nr02216a knowledge, only one experiment has been reported showing simultaneous current and direct current noise mapping with spatial resolution. ${ }^{25}$

However, this experiment did not study the shot noise regime but concentrated on $f^{-\beta}$ noise contributions in the power spectral density, where $f$ is the frequency and $\beta$ is an empirical parameter close to one. Such extrinsic noise is usually dominant and hampers the use of noise measurements in STM investigations to reveal intrinsic features caused by e.g. electronic correlations. In general several noise contributions exist, arising from the various components of the circuit. These components may vary significantly when varying, e.g., the current while scanning a corrugated surface, erroneously mimicking features in the noise signal.

For demonstrating the successful operation of our novel scheme we concentrate here on the intrinsic fundamental noise due to the tunnelling effect, i.e. a system in which the noise is expected to be white, spatially constant, and its size can be precisely computed from fundamental theory without free parameters.

For this purpose we produce defect structures with small lateral size that serve as test structures for comparing the constant current images with the noise images. At first we show that the topography images can quantitatively be described, when taking into account the non-exponential radial distribution of the tunnelling current. This quantitative description enables us to ascribe the observed ultrasmall defect structures to arise from tunnelling between d-states in the tip and in the sample. In the next step we record and analyse the noise while scanning these topographically pronounced structures and verify that the noise map is featureless, despite the strong corrugation in the topography. 


\section{Quantum noise measurements}

\subsection{Theoretical background}

For a tunnel barrier with transmissions $T_{\mathrm{n}} \ll 1$ of currentcarrying conduction channels, involving only elastic processes at the barrier, and at voltages where the density of states can be considered constant, statistical considerations result in a lowfrequency limit of the current noise power spectral density

$$
S=2 e V G_{0} \operatorname{coth}\left(\frac{\mathrm{eV}}{2 k_{\mathrm{B}} T}\right) \sum_{n} T_{\mathrm{n}}
$$

with the conductance quantum $G_{0}=2 e^{2} / h$. Here, $k_{\mathrm{B}}$ is Boltzmann's constant, $V$ is the applied voltage, $e$ is the elementary charge, $h$ is Planck's constant, and $T$ is the absolute temperature. ${ }^{2}$ When the voltage exceeds the thermal energy $k_{\mathrm{B}} T / e$, expression (1) exponentially approaches the quantum limit $2 e|I|$, where $I$ is the DC component of the tunnelling current (TC). Interactions in the transport result in deviations from this simple relationship that are usually accounted for by the Fano factor $F$ with

$$
S=2 e|I| F
$$

as shown e.g. in ref. 2-4 for highly correlated quasiparticles in two-dimensional electron gases or superconducting point contacts, respectively. We present here a novel and simple noise measurement scheme that enables us to demonstrate $F=1$ at all positions for a model system in which the transport takes place through particular atomic orbitals despite a pronounced topography in the current image. This finding is important in particular for the field of molecular electronics where local deviations from bare tunnelling might be expected.

\subsection{Design of the experiment}

We probe the current noise in a home-made low-temperature temperature scanning tunnelling microscope (STM) with very high thermal, mechanical and electronic stability. ${ }^{26}$ The Ir (111) single crystal was thoroughly cleaned as described in the ESI. $\dagger$ The clean Ir (111) sample was immediately installed on the sample holder of the STM. At room temperature, the vacuum can hosting the STM with a knife-cut Ir tip is pumped to a moderate vacuum of $p=10^{-3}$ Pa. During cool-down the temperature to approximately $30 \mathrm{~K}$, the sample and the tip are steadily kept several Kelvin above the coldest parts of the insert for limiting condensation of contaminants. The electronic experiments are then performed at $4.2 \mathrm{~K}$ in a cryogenic vacuum in the order of $10^{-11} \mathrm{~Pa}$ or lower. The electronic measurement setup is modified for extracting the current noise and its spectral density without the necessity of double wiring and performing cross-correlation ${ }^{7}$ and without going to high frequency measurements. ${ }^{27}$ The AC-coupled current output signal of a lownoise current-to-voltage converter (IVC) is sent through a setup very similar to that described in ref. 25 or 28 , containing a fourth order band pass with a centre frequency of $1.5 \mathrm{kHz}$ and a quality factor of six. In the linear range of the amplifier, the band-limited noise signal is squared and averaged, to obtain the direct analogue of the total noise power, which is

$$
V_{\text {Noise, }, \text { ontact }}=g^{2} \int_{0}^{\infty}\left(S+S_{0}(f)\right) w(f) \mathrm{d} f=V_{\text {Noise }, S}+V_{\text {Noise }, 0}
$$

including the white noise $S$ of the tunnelling contact, resulting in the voltage noise contribution $V_{\text {Noise }, S}$ and the amplifier noise $S_{0}(f)$ resulting in the voltage noise contribution $V_{\text {Noise, } 0}$. The tip-sample capacitance is usually low enough to allow for measuring the uncorrelated offset noise $V_{\text {Noise, } 0}$ when retracting the tip. The function $w(f)$ ( $f$ being the frequency) has a roll-off of order eight above and below the pass-band, and $g$ is an effective gain factor of the amplifier chain.

By subtracting the offset voltage noise contribution, $V_{\text {Noise, } 0}$, we obtain the tunnelling contact noise power spectral density

$$
S=\left(V_{\text {Noise }, \text { contact }}-V_{\text {Noise }, 0}\right) /\left(g^{2} \int_{0}^{\infty} w(f) \mathrm{d} f\right)
$$

The noise measurement system is calibrated by measuring the output change produced by the Johnson current-noise power spectral density $4 k_{\mathrm{B}} T / R$ of a resistor $R$ with very low capacitance at the input, substituting the sample, and the IVC noise was further checked to be independent of the DC current through reference resistors. Further details of the schematic diagram, examples of noise spectra and breakdown of major detector noise components, calibration of the analogue noise signal, etc. are presented in the ESI. $\dagger$

To avoid any variable perturbations of the noise signal that could be caused by vibrations in the tunnelling barrier or fluctuations of the tunnelling voltage, the tunnelling voltage and the piezoelectric actuator voltages are low-pass filtered when the setup is switched into the noise-acquisition mode. The bandwidth of the STM feedback loop is kept well below the relevant cut-off frequency of the actuator system. The IVC includes a linear range and a logarithmic range described in ref. 29. The measurements are performed in the linear range, and the contacts are closed in the logarithmic range.

\section{Results and discussion}

\subsection{Fabrication of local defects}

To produce surface structures with spatial variations of subatomic size - from here on labeled "subatomic features" - the sample surface is modified by repeatedly impinging the tip into the sample surface and thus forming a metallic point contact, employing a dynamic STM mode similar to that described in ref. 30 at average currents in the range of up to $1 \mathrm{~mA}$, voltages up to 0.5 Volts, and tapping frequencies between $40 \mathrm{~Hz}$ and $120 \mathrm{~Hz}$. Excitation is performed by applying an AC voltage to the longitudinal channel of the scanner piezo. The number of conductance quanta reached at the lower turning point can be observed directly at the oscilloscope for up to $\sim 10 G_{0}$. This procedure has been applied successfully to form clean atomic contacts and atomic chains of Ir and other metals despite the presence of residual contaminants on the surface. ${ }^{31}$ After the described modification procedure the STM is operated at a conductance $<10^{-3} G_{0}$ and voltages $<100 \mathrm{mV}$. Typical constant current images exhibit defect structures at variable scales down 
to subatomic dimensions as depicted in Fig. 1. Features at atomic and smaller scales are clearly visible.

\subsection{Topography}

In this section we briefly address the origin of the highly corrugated structures. Subatomic resolution in scanning probe techniques is a fascinating field by itself. Subatomic details in STM imaging have been quantitatively described in the pioneering work of Chen, ${ }^{32}$ taking into account orbital convolutions. Features of subatomic size observed by atomic force microscopy under UHV conditions and ascribed to p orbitals have been reported earlier for a Si surface ${ }^{33}$ and recently by STM when using a tip with $\mathrm{p}$ electronic character. ${ }^{34}$ Since our main interest in these structures is to use them as test bed for qualifying the noise measurement scheme, we keep this section concise and give further details in the ESI. $\dagger$

The subatomic features occur regularly after typically $10^{2}$ to $10^{4}$ indentations, however, with variable shape and stability reflecting that they present metastable states with limited lifetime. After several hours or repeated scanning with high currents they may disappear again or change their spatial appearance on the subatomic scale while the neighbourhood
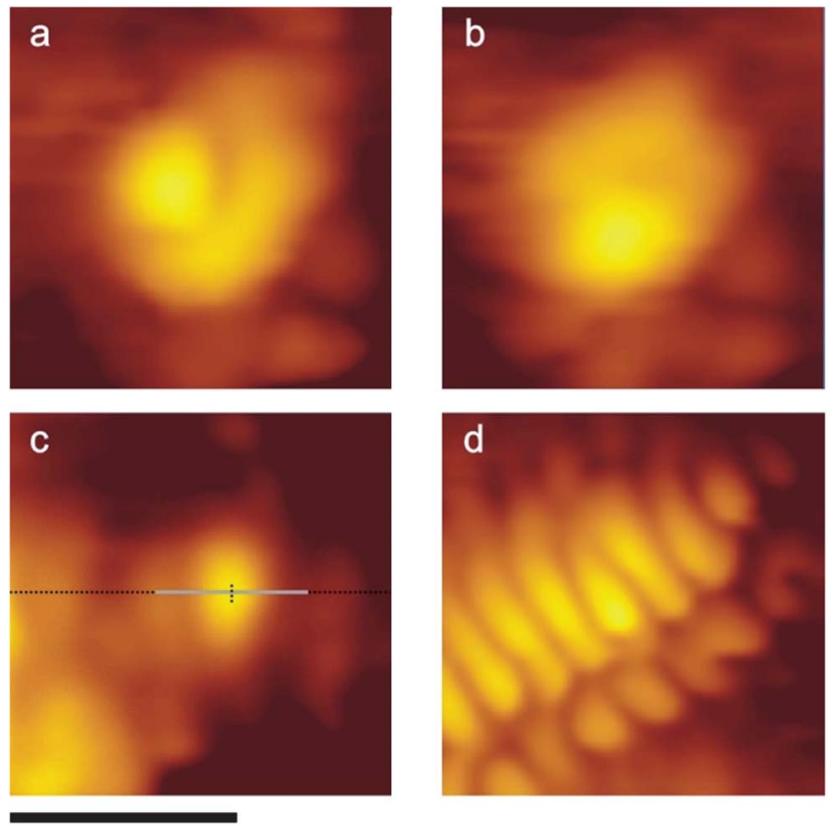

$1 \mathrm{~nm}$

Fig. 1 Constant current images obtained nearby the location where an Ir contact has been formed in the dynamic STM mode with intermittent contact. The vertical ranges of the topographic areas are (a) $0.66 \mathrm{~nm}$, (b) $0.65 \mathrm{~nm}$, (c) $0.46 \mathrm{~nm}$, and (d) $1.10 \mathrm{~nm}$. A flip of the electronic structure occurs spontaneously, transferring the configuration shown in (a) to the configuration in (b). Remarkably, the background topographic feature at the right lower corner of the images (a) and (b) does not change considerably while the subatomic structures at the centre do change. See the ESI† document for an apical cross-section of topography (c) along the horizontal dotted line. The grey area marks a region where extended noise probing has been performed (see Fig. 3). The rim-like structures in frames (c) and (d) are interpreted as interacting d-electronic tip and sample states of Ir. ${ }^{29,30}$ Tunnelling parameters: (a) $V=11.5 \mathrm{mV}, I=190 \mathrm{pA}$, (b) $V=11.5 \mathrm{mV}$, $I=103 \mathrm{pA}$, (c) $V=11.3 \mathrm{mV}, I=109 \mathrm{pA}$, and (d) $V=32.4 \mathrm{mV}, I=257 \mathrm{pA}$. topography remains constant. For example the structures in the lower right corner of Fig. 1(a) and (b) are unchanged although the subatomic features in the centre are different. This observation points to an electronic (orbital) nature of the structures. Given the very low temperature at which the experiment is run, we believe that surface diffusion of the atoms can be excluded. However, the defect structures most likely represent metastable states including electronic trap states. We assume that the relaxation of the defect states causes the time-dependence of the subatomic features, e.g. by dislocations of the subatomic distance of atoms in the surface or the tip that lead to a change of the overlap of the wave functions.

In frame Fig. 1(a), the curvature radius of the symmetrical central peak is $180 \mathrm{pm}$, corresponding to the convolution matrix elements of higher angular momentum electronic states of Ir. ${ }^{30,32}$ Accordingly, the structures in frames (a) and (b) are interpreted as Ir d-states imaged by a state of higher symmetry, presumably an s-state of the tip. We note that the roles of the "tip" and the "sample" are interchangeable in STM experiments. Since the typical curvature radii close to the topographic maxima in frames (c) and (d) are ranging down to $\sim 90 \mathrm{pm}$, presumably higher angular momentum (d-) states are present on both sides of the junction, yielding extremely small apex radii and more complex structures. In frame (d), we measure a distance of $(223 \pm 12) \mathrm{pm}$ between the fins of the corrugated structure, corresponding well to the Ir atomic double bond covalent distance of 230 pm. ${ }^{35}$

In the ESI $\dagger$ we justify our interpretation by a detailed quantitative analysis. We show that they are described quantitatively by the full tunnelling theory and how the quantitative understanding can be used for determining the real distance between the closest nuclei. The narrow structures are in agreement with the expected shape arising from the overlap of higher angular momentum ( $l=2$ or higher) states with sharp lobes of the wave functions. $^{30,32}$ We demonstrate that in STM the geometric effects of orbital interactions play an important role. We believe that our work represents the first demonstration of spatially resolved d-orbitals in a metallic system by scanning tunnelling microscopy.

\subsection{Shot noise mapping}

We now turn to the noise measurements. At the position of the topographic maximum shown in Fig. 1(c) and at a tunnelling resistance of $\sim 37 \mathrm{M} \Omega$, the STM feedback was switched off and simultaneous noise and current $(S / I / V)$ spectroscopy was performed, $c f$. Fig. 2.

The non-contact offset noise-density was determined at the retracted tip and subtracted from the data. Besides a small additional offset, the measured noise follows to a good approximation the quantum limit $2 e|I|$ above the thermal energy, which is in the order of $1 \mathrm{meV}$. Deviations can be caused by an increased tip-sample capacitance that has an influence on the noise offset. The linear dependence of the noise power on the current above the thermal energy is a signature of the shot noise. Any linear cross-talk of a disturbance into the TC, or $1 / f$ contributions, would produce quadratic components. ${ }^{5}$ The 


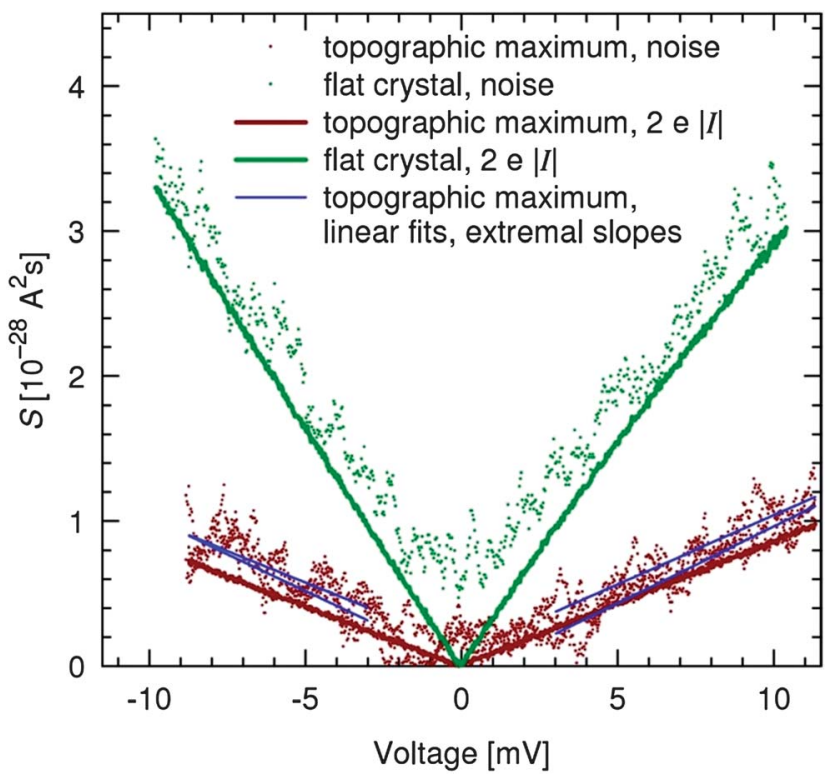

Fig. 2 S/IIV spectroscopy at the position of the topographic maximum of Fig. 1(c) at a tunnelling resistance of $37 \mathrm{M} \Omega$, corresponding to $308 \mathrm{pA}$ at $11.5 \mathrm{mV}$, and above a flat location at $\sim 10 \mathrm{M} \Omega$. Above the thermal energy, which is in the range of $1 \mathrm{meV}$, the measured noise is slightly above the limiting scale $2 \mathrm{e} \mid /$. Current offset, voltage offsets and nominal non-contact noise offset have been subtracted. The noise power is given by the average of measurements that are acquired during a measurement period in which no other instabilities are observed. The blue lines symbolize extremal slopes of $S(V)$ dependences that are compatible with the measured noise.

residual variations are mainly determined by detector noise. This observation suggests that at the peak of the current image marked in Fig. 1(c), the Fano factor is close to one, and the tunnelling is mainly determined by the statistical transition of electrons given by the quantum mechanical transmissions $T_{\mathrm{n}}{ }^{2}$ A numerical fit for the Fano factor above the thermal energy, allowing for linear effects in $S(V)$, has been performed for $|V|>$ $3 \mathrm{mV}$. The resulting Fano factors are in the range $1.04<F<1.22$ for negative voltages and $1.11<F<1.24$ for positive voltages. The four extremal results of six fittings belonging to three voltage sweeps are shown in Fig. 2. The standard deviations of $F$ are in the range of 0.07 for negative $V$ and 0.03 for positive $V$.

The largest experimental error is the detector noise determining the uncertainty of the single measurements shown in Fig. 2. However, it is possible to reduce this statistical error to the limits given by the stability of the measurement setup and all other possible residual errors. Residual errors and their properties are discussed in the ESI. $\dagger$ The detector noise shrinks dramatically when averaging over many measurements in an averaging fit, and the Fano factor is close to one.

Within the experimental accuracy, the Fano factor was not observed to fall below $F=1$, which is expected for uncorrelated electronic transport when no double-barriers are present. ${ }^{5}$ An increased noise is consistent with theories including excitations of phonons in the mesoscopic tunnelling regime. ${ }^{8-15}$ However, the current should increase by the same ratio, so that the Fano factor would not deviate from $F=1$ in this limit. In our experiment deviations can be caused by an increased tip-sample capacitance that influences the noise offset. Fig. 3 shows a simultaneous noise and topographic mapping above the grey area marked in Fig. 1(c), recorded at a tunnelling resistance of $77 \mathrm{M} \Omega$. The topographic image of the electronic structure is slightly different from the images shown in Fig. 1 and crosssections presented in the ESI. $\dagger$ Parts of the changes are caused by sensitivity with respect to the vertical current gradient due to reduced topographic controller gains. A Fano factor close to one with no significant spatial contrast is observed. The residual fluctuation is mainly determined by detector noise. In line with the above discussion of the $S(V)$ error, the Fano factor error shrinks considerably by averaging this detector-driven error over several scan lines. This is directly visualized in Fig. 3. The rms-deviation of the determined Fano factor $F=1.13 \pm 0.11$ when averaging over five scan-lines is in the range expected from dividing the rms-deviation of $F=1.13 \pm 0.18$ determined from the pixels of five scan-lines by a factor of $\sqrt{5}$. Possible sources of systematic deviations changing the statistical properties are a slight increase at the borders of the in-plane scan range caused by changing the input capacitance due to a highly effective tip radius and changing the tip-sample distance, and the spectral weight in different frequency ranges. Spot tests of $S / I / V$ spectroscopy, time spectra, scanning and recording the noise signal have been performed above several locations of the images shown in Fig. 1, and others. Time spectra confirm white

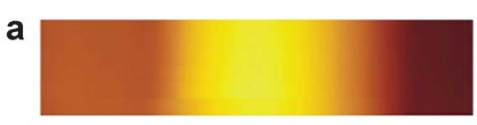

b
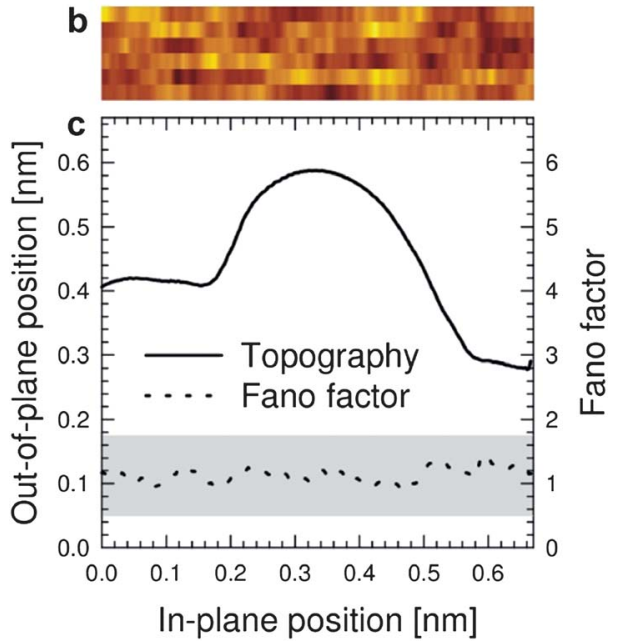

Fig. 3 Simultaneous high resolution imaging of topography and Fano factor. (a) Topographic image contrast covering the grey area of Fig. 1(c), raw data. Only a small area is scanned because of the slow noise measurement speed that is required to obtain stable imaging with the piezo filtering engaged, and to obtain sufficient resolution in the noise measurement, see ESI. $\uparrow$ The scale of the slow scanning direction has been magnified by a factor of 10 . Scan direction from right to left. Tunnelling resistance: $77 \mathrm{M} \Omega$. (b) Corresponding noise contrast with offset correction. Brighter areas correspond to lower noise. (c) Cross-section of topography along the first scan line of frame (a), raw data, and cross-section of average Fano factor $F$, averaged over five scan lines. The lowered integrator part of the feedback loop causes a current increase at the image start. Scan speed: $42 \mathrm{pm} \mathrm{s}^{-1}$. Imaging parameters: $11.5 \mathrm{mV}, 150 \mathrm{pA}$. The brightness range in (b) covers $0.5<$ $F<1.75$ and is represented by the borders of the grey area in (c). 
noise within the measurement accuracy. The Fano factor $F=1$ is observed to be a lower limit for the current noise, and the $I / V$ curves are linear within the experimental accuracy. Further noise analysis is given in the ESI. $\uparrow$ Within the measurement accuracy, the noise power is - as theoretically expected - not correlated with the topographic signal, proving the excellent performance of the noise measurement scheme.

\section{Conclusions}

In conclusion, we used a simple method to produce features with subatomic spatial variation showing all the geometry expected for sd-hybrid orbital systems. These structures are used to demonstrate the functionality of a novel noise measurement scheme that does not require double wiring and cross-correlation, but can be performed with a standard commercial STM electronic setup. Careful modelling and calibration procedures are presented that enable quantitative determination of the shot noise of tunnelling currents. Simultaneous probing of topography and noise leads to the conclusion that "statistical" tunnelling is dominant when imaging the detailed real space structures. The linear relationship between noise and current is robust within the sub-atomic details, and above the thermal energy. The measured current noise is not influenced by the constant-current topography. Statistical tunnelling indicates that very probably no resonant oscillatory process occurs in the constant current imaging. In the case of a highly oscillatory dynamic process correlated with the tunnelling, the noise is expected to differ significantly from $F=1$, and a correlation with the topographic controller output would be expected. We thus confirm that the observed topographic structures are determined by elastic tunnelling. Spatially dependent noise might be expected if electronic correlations are relevant for the electronic transport, for e.g. inelastic cotunnelling effects, ${ }^{36}$ Kondo correlations ${ }^{37}$ or molecular orbital coupling. ${ }^{38}$ Our results pave the way to high-resolution noise mapping of molecular and other nanostructures on surfaces.

\section{Acknowledgements}

We gratefully acknowledge discussions with M. Wolz, O. Tal, and F. Haupt and financial support from Deutsche Forschungsgemeinschaft through Collaborative Research Center SFB767.

\section{References}

1 Y. V. Nazarov, Quantum Noise in Mesoscopic Physics, Springer, Berlin, Germany, 2003.

2 Ya. M. Blanter and M. Büttiker, Phys. Rep., 2000, 336, 1.

3 L. Saminadayar, D. C. Glattli, Y. Jin and B. Etienne, Phys. Rev. Lett., 1997, 79, 2526.

4 M. Reznikov, R. de Picciotto, T. G. Griffiths, M. Heiblum and V. Umansky, Nature, 1999, 399, 238.

5 H. Birk, M. J. M. de Jongh and C. Schönenberger, Phys. Rev. Lett., 1995, 75, 1610.

6 R. Cron, M. F. Goffman, D. Esteve and C. Urbina, Phys. Rev. Lett., 2001, 86, 4104.
7 B. Ludoph, M. H. Devoret, D. Esteve, C. Urbina and J. M. van Ruitenbeek, Phys. Rev. Lett., 1999, 82, 1530.

8 F. Haupt, F. T. Novotny and W. Belzig, Phys. Rev. Lett., 2009, 103, 136601.

9 F. Haupt, T. Novotny and W. Belzig, Phys. Rev. B: Condens. Matter Mater. Phys., 2010, 82, 165441.

10 T. Novotny, F. Haupt and W. Belzig, Phys. Rev. B: Condens. Matter Mater. Phys., 2011, 84, 113107.

11 T. L. Schmidt and A. Komnik, Phys. Rev. B: Condens. Matter Mater. Phys., 2009, 80, 041307.

12 R. Avriller and A. Levy Yeyati, Phys. Rev. B: Condens. Matter Mater. Phys., 2009, 80, 041309.

13 D. F. Urban, R. Avriller and A. Levy Yeyati, Phys. Rev. B: Condens. Matter Mater. Phys., 2010, 82, 121414.

14 Y. Utsumi, O. Entin-Wohlman and A. Ueda Aharony, Phys. Rev. B: Condens. Matter Mater. Phys., 2013, 87, 115407.

15 M. Kumar, R. Avriller, A. Levy Yeyati and J. M. van Ruitenbeek, Phys. Rev. Lett., 2012, 108, 146602.

16 R. Deblock, E. Onac, L. Gurevich and L. Kouwenhoven, Science, 2003, 301, 203.

17 L. Spietz, K. Lehnert, I. Siddiqi and R. Schoelkopf, Science, 2003, 300, 1929.

18 B. Reulet, J. Senzier and D. Prober, Phys. Rev. Lett., 2003, 91, 196601.

19 R. Schleser, E. Ruh, T. Ihn, K. Ensslin, D. C. Driscoll and A. C. Gossard, Appl. Phys. Lett., 2004, 85, 2005.

20 T. Fujisawa, T. Hayashi, Y. Hirayama, H. D. Cheong and Y. H. Jeong, Appl. Phys. Lett., 2004, 84, 2343.

21 J. Bylander, T. Duty and P. Delsing, Nature, 2005, 434, 361.

22 C. Flindt, C. Fricke, F. Hohls, T. Novotny, K. Netocny, T. Brandes and R. J. Haug, Universal oscillations in counting statistics, Proc. Natl. Acad. Sci. U. S. A., 2009, 106, 10116.

23 N. L. Schneider, G. Schull and R. Berndt, Phys. Rev. Lett., 2010, 105, 026601.

24 N. L. Schneider, J. T. Lü, M. Brandbyge and R. Berndt, Phys. Rev. Lett., 2012, 109, 186601.

25 B. Koslowski, C. Baur, R. Möller and K. Dransfeld, Surf. Sci., 1993, 280, 106.

26 C. Debuschewitz, F. Münstermann, V. Kunej and E. Scheer, J. Low Temp. Phys., 2007, 147, 525.

27 R. Chen, P. J. Wheeler and D. Natelson, Phys. Rev. B: Condens. Matter Mater. Phys., 2012, 85, 235455.

28 D. van Baak, Noise Fundamentals, NF1-A, instructors manual, Teachspin, Inc., 2010.

29 U. Dürig, L. Novotny, B. Michel and A. Stalder, Rev. Sci. Instrum., 1997, 68, 3814.

30 M. Herz, F. J. Giessibl and J. Mannhart, Phys. Rev. B: Condens. Matter Mater. Phys., 2003, 68, 045301.

31 N. Agrait, A. Levy Yeyati and J. M. van Ruitenbeek, Phys. Rep., 2003, 377, 81.

32 C. J. Chen, Introduction to Scanning Tunneling Microscopy, Oxford University Press: Oxford, New York, USA, 1993.

33 F. J. Giessibl, S. Hembacher, H. Bielefeldt and J. Mannhart, Science, 2000, 289, 422.

34 L. Gross, N. Moll, F. Mohn, A. Curioni, G. Meyer, F. Hanke and M. Persson, Phys. Rev. Lett., 2011, 107, 086101. 
35 P. Pyykkö and M. Atsumi, Chem.-Eur. J., 2009, 15, 12770.

36 H. van Houten, C. W. J. Beenakker and A. A. M. Staring, in Single Charge Tunneling: Coulomb-Blockade Phenomena in Nanostructures, Plenum Press and NATO Scientific Affairs Division, 1992, p. 167.
37 J. Bork, Y.-H. Zhang, L. Diekhöner, L. Borda, P. Simon, J. Kroha, P. Wahl and K. Kern, Nat. Phys., 2011, 7, 901.

38 J. Repp, G. Meyer, F. Olsson and M. Persson, Controlling the charge state of individual gold adatoms, Science, 2004, 305, 493. 